\title{
Rotorcraft Waypoint Guidance Design Using SDRE Controller
}

\author{
Chang Deok Yang* \\ Aero Propulsion System Department, Korea Aerospace Research Institute
}

\section{Chang-Joo Kim**}

Department of Aerospace Information Engineering, Konkuk University, Seoul, Korea

Sooseok Yang***

Aero Propulsion System Department, Korea Aerospace Research Institute

\begin{abstract}
This paper deals with the State-Dependent Riccati Equation (SDRE) Technique for the design of rotorcraft waypoint guidance. To generate the flight trajectory through multiple waypoints, we use the trigonometric spline. The controller design and its validation is based upon a level 2 simulation rotorcraft model and the designed SDRE controller is applied to the trajectory tracking problems. To verify the designed guidance law, the simulation environment of high fidelity rotorcraft model is developed using three independent PCs. This paper focuses on the validation of rotorcraft waypoint guidance law which is designed by using SDRE Controller.
\end{abstract}

Key words : Rotorcraft Flight Control, SDRE, Trajectory Generation, Waypoint Guidance

\section{Introduction}

The State-Dependent Riccati Equation (SDRE) technique is based on the Linear Quadratic Regulator (LQR) method and it allows us to design the controllers for the nonlinear system with the same technique used in its linear counterparts [1,2]. The SDRE technique basically differs from the LQR method in two points. First, the SDRE technique demands a linear system-like structure with State-Dependent Coefficients (SDC) form of equation of motion. But the general multi-variable nonlinear systems like rotorcraft do not always conform to this structure. Also, it is well known that the SDC form for multivariable systems is not unique and there is no general method for its derivation. Second, the LQR method requires off-line solution of the Algebraic Riccati Equation (ARE). However, to calculate the feedback control in on-line, the SDRE method needs the iterative solution of the ARE because the state and control derivative matrices of derived SDC form are generally time-varying. Therefore an efficient numerical solution of the Algebraic Riccati Equation (ARE) is crucial for the system performance.

\footnotetext{
* Graduate Student

** Professor

E-mail : cjkim@konkuk.ac.kr Tel : +82-2-450-3547 Fax : +82-2-444-6670 *** Director
} 
Langson et. al. [3] presented the experimental implementation of the SDRE controller for a third order nonlinear system. In its setup, one $75 \mathrm{MHz}$ Pentium computer was used to simulate the plant dynamics, and design the SDRE controller with $100 \mathrm{~Hz}$ sampling rate. P. K. Menon et. al. [4] applied the SDRE controller to the missile control. To solve the ARE, various numerical methods and $\mathrm{PC}$ processors are used with $20 \mathrm{kHz}$ sampling rate. Above studies were made it possible to implement of SDRE in the real-time because comparatively simple models were used. However, the SDRE technique demands high computing power when it is applied to complex dynamic models. In this regard, Kim et. al. [5] applied the indirect method to the various rotorcraft model to investigate the relative computational burden. In the Ref. [6] Bogdanov et. al. applied the SDRE technique to the small scale rotorcraft and they represented its flight test in hovering and low flight speed region.

The design of flight path for unmanned aircraft is one of the important issues. It should be shortest path in time and satisfied with the aircraft dynamics. In the Ref. [7], Beard et. al. design the flight path using voronoi diagram. This method doesn' $t$ require the high computation but it needs a post-processing to convert waypoints to flight path. Bakaric et. al. [8] suggest the line of sight with heading correction method. They applied it to the underwater vehicle having simple dynamics with low forward speed. Nagi [9] and Rodrigues [10] present the trigonometric interpolating curve to generate the trajectory through multiple waypoints. This curve is obtained by putting together smaller pieces (spline segments). Since each piece can be computed separately, it can cope with unpredictable changes of waypoint, like the appearance of sudden obstacles. And one may reduce the computation by selecting the proper time interval.

Present study uses the trigonometric interpolating curve to generate flight path through multiple waypoints. And we apply designed flight path to the trajectory tracking controller using SDRE technique. The SDRE controller is verified on the simulation environment through waypoint guidance simulation. The level 2 simulation rotorcraft model [11] is used to design and verify the SDRE controller.

\section{SDRE Technique}

\subsection{SDRE Controller Design}

The general infinite-horizon nonlinear regulator problem can be represented as:

$$
\begin{aligned}
& \min J(x, u, t)=\frac{1}{2} \int_{t_{0}}^{\infty} x(t)^{T} Q x(t)+u(t)^{T} R u(t) d t \\
& \text { s.t } \dot{x}=f(x)+B(x) u
\end{aligned}
$$

where, $Q \geq 0, R>0$

The SDRE technique requires the SDC form as shown in Eq. (3) with which the SDRE method finds controller gain by assuming the matrices, $A(x)$ and $B(x)$, are locally constant and by solving the ARE.

$$
\dot{x}=A(x) x+B(x) u
$$

If $\{A(x), B(x)\}$ in Eq. (3) is a controllable pair, the procedure of the SDRE technique is summarized as follows.

(i ) Drive the SDC form as show in Eq. (3) using a SDC factorization.

( ii ) Solve the State-Dependent Algebraic Riccati Equation (SDRE) to obtain $P(t) \geq 0$, where $P$ is function of $x$

$$
P A(x)+A^{T}(x) P-P B(x) R^{-1} B^{T}(x) P+Q=0
$$

( iii ) Construct the nonlinear feedback controller

$$
u=-K x(t)=-R^{-1} B^{T} P x(t)
$$


On the contrast to the LQR solution, the SDRE technique repeatedly requires the SDC factorization and the solution of state-dependent ARE at each time of control engagement. Therefore, the performance of SDRE controller depends on the derivation of SDC form and efficient solution of ARE.

A unified approach to obtain the SDC factorization for a general nonlinear system is impossible because the SDC form for a multivariable system is not unique [2]. Any function, $f(x) \in C^{1}$, can be expressed as in the form of Eq. (6) but the condition represented in Eq. (7) should be meet to reduce a dynamic system to the SDC form.

$$
\begin{aligned}
& f(x)=A(x) x+\Delta f(x) \\
& \lim _{x \rightarrow 0} f(x) / x \leq M<\infty
\end{aligned}
$$

And in case, the system dynamics of control-affine system can be factorized into two different ways, any combination of these would generate a SDC form. But because the control inputs are calculated when the stability is guaranteed, the matrix A, B cannot be selected randomly.

The numerical efficiency in solving the ARE in Eq. (4) is crucial especially for online implementation of the SDRE controller. In the case of the rotorcraft, the dynamic system is inherently unstable. Therefore the numerical solution of the Lyapunov equation needs the initial estimation if the gain matrix $K$ with which the resultant system matrix $A-B K$ becomes Hurwitz.

\subsection{Numerical Methods}

In the previous research [6], A. Bogdanov et. al. proposed a numerical SDC factorization method for nonlinear rotorcraft dynamic model. This paper uses the modified version of the SDC factorization proposed by Kim et. al. [2], by considering both system equilibrium states and kinematical offset in forcing functions. The nonlinear equation of motion at an arbitrary time instant and that at an equilibrium condition can be written as following.

$$
\begin{gathered}
\dot{x}=F(x, u) \\
\dot{x}_{0}=F\left(x_{0}, u_{0}\right)=\text { const }
\end{gathered}
$$

Where, $x_{0}, u_{0}$ denote equilibrium states and controls, respectively.

Even in an equilibrium state, the value of forcing function, $F\left(x_{0}, u_{0}\right)$, is generally nonzero. The consideration on the state variation from the equilibrium condition and the Taylor series expansion of motion equation around the equilibrium states and controls enable us to derive the motion equation for the perturbed states and controls as shown in Eq. (12).

$$
\begin{aligned}
& x=x_{0}+\tilde{x}, \quad u=u_{0}+\tilde{u} \\
\dot{x} & =\dot{x}_{0}+\dot{\tilde{x}} \\
& =F(x, u) \\
& =F\left(x_{0}, u_{0}\right)+\left(\nabla_{x} F\right) \tilde{x}+\left(\nabla_{u} F\right) \tilde{u}+\operatorname{HOT}(\tilde{x}, \tilde{u}) \\
\dot{\tilde{x}} & =F(x, u)-F\left(x_{0}, u_{0}\right) \\
& =\left(\nabla_{x} F\right) \tilde{x}+\left(\nabla_{u} F\right) \tilde{u}+\operatorname{HOT}(\tilde{x}, \tilde{u})
\end{aligned}
$$

Where, $\operatorname{HOT}(\tilde{x}, \tilde{u})$ is high order term in $\tilde{x}$ and $\tilde{u}$.

As previous mentioned, an SDC form can be derived when the higher order term is a function of state variables only as in a control affine system like Eq. (2)

Then, the SDC factorization can be carried out by using the following steps $[2,12]$. 
( i ) Control derivative matrix

$$
B(x)=B(\tilde{x})=\frac{\partial \tilde{F}}{\partial \tilde{u}},
$$

Where, $\tilde{F}(x, u)=F(x, u)-F\left(x_{0}, u_{0}\right)$

(ii) State derivative matrix

Where, $\tilde{f}(\tilde{x})=\tilde{F}(x, u)-B(x) \tilde{u}$

$$
\tilde{f}(\tilde{x})=A(\tilde{x}) \tilde{x},
$$

The control derivative matrix $B(\tilde{x})$ can be approximated using a finite difference method. And the state derivative matrix $A(\tilde{x})$ can be computed using function values around $\tilde{x}$. If we use $2 \mathrm{~N}$-points around $\tilde{x} \in R^{N}$, the matrix can be computed using the following formula.

$$
\tilde{A}(\tilde{x})=F X^{T}\left(X X^{T}\right)^{-1}
$$

$$
\text { Where, } \begin{aligned}
& F=\left[\tilde{f}\left(\zeta_{1}\right), \tilde{f}\left(\zeta_{1}^{-}\right), \cdots, \tilde{f}\left(\zeta_{n}\right), \tilde{f}\left(\zeta_{n}^{-}\right)\right] \\
& X=\left[\zeta_{1}, \zeta_{1}^{-}, \cdots, \zeta_{n}, \zeta_{n}^{-}\right] \\
& \tilde{f}\left(\zeta_{1}\right)=\tilde{A}(\tilde{x}) \zeta_{1} \quad \text { with } \zeta_{1}=\tilde{x}+\sigma_{1} \\
& \tilde{f}\left(\zeta_{1}^{-}\right)=\tilde{A}(\tilde{x}) \zeta_{1}^{-} \quad \text { with } \zeta_{1}^{-}=\tilde{x}-\sigma_{1} \\
& \vdots \\
& \tilde{f}\left(\zeta_{n}\right)=\tilde{A}(\tilde{x}) \zeta_{n} \quad \text { with } \zeta_{n}=\tilde{x}+\sigma_{n} \\
& \tilde{f}\left(\zeta_{n}^{-}\right)=\tilde{A}(\tilde{x}) \zeta_{n}^{-} \text {with } \zeta_{n}^{-}=\tilde{x}-\sigma_{n}
\end{aligned}
$$

As mentioned in previous section, the SDRE technique requires the derivation of the SDC form of motion equations and the solution of the state-dependent ARE. There are the causes of high computation burden. Many numerical methods to solve the ARE are being developed but the selection from the existing one is not simple since the efficiency and applicability of each algorithms depend on numerous factors.

H.T Bank and K. Ito proposed a hybrid method to solve the ARE for a system with a large size [13]. In their method, the initial feedback gain $K$ is obtained from the limiting solution of Chandrasekhar system and the result is refined through the Newton-Kleinman algorithm. Since the system dynamics of the rotorcraft are inherently unstable, the initial feedback system can be unstable. The solution of the Chandrasekhar system provides a stable feedback gain matrix with suitable chosen weighting matrices even for an unstable system.

The Chandrasekhar system for initial gain can be written as following and the suitable time integrator such as Runge-Kutta algorithm can be used for resolving the initial value problem.

$$
\left[\begin{array}{c}
\dot{K}(t) \\
\dot{L}(t)
\end{array}\right]=\left[\begin{array}{c}
-B^{T} L^{T}(t) L(t) \\
-L(t)[A-B K(t)]
\end{array}\right] \text { with }\left[\begin{array}{c}
K(0)=0 \\
L(0)=C=Q^{1 / 2}
\end{array}\right]
$$

Where, $A$ and $B$ are the state derivative matrix and control derivative matrix of SDC form, respectively.

The state-dependent ARE shown in Eq. (4) can be solved by applying the NewtonKleinman algorithm for following transformed system [2, 12].

$$
\begin{aligned}
& S=A-B K \\
& S^{T} P+P S+K^{T} R K+Q=0
\end{aligned}
$$


If we obtain a stable pair $\left\{A-B K_{0}, B\right\}$ by solving Chandrasekhar system, the control gain matrix is iteratively obtained by applying the Newton-Kleinman algorithm using the following equations $\left(i=1, \cdots, i_{\max }\right)$.

$$
\begin{gathered}
S_{i}=A-B K_{i} \\
S_{i}^{T} P_{i}+P_{i} S_{i}=-Q-K_{i}^{T} R K_{i} \\
\lim _{i \rightarrow \infty} P_{i}=P
\end{gathered}
$$

where, $K_{i+1}=R^{-1} B^{T} P_{i}$

\section{Waypoint Guidance Design}

\subsection{Rotorcraft Model}

The rotorcraft flight dynamic model used in this study is based on the previous research in Ref. [11]. Since the flap and inflow states are the unobservable states that cannot measured or estimated, these states are considered as hidden or unobservable states during the controller design [2,12]. Therefore, the rotorcraft model for controller design is defined by using the rigid body states as shown in Eq. (20) and the rotor states corresponding to flap motion and inflow are estimated by using the rotor trim solution. However, the level 2 simulation model developed in Ref. [11] is used as the validation model.

$$
x_{R}=\left[u, v, w, p, q, r, \phi, \theta, \psi, x_{E}, y_{N}, h\right]
$$

\subsection{Trajectory Planning}

In this work, we use the trigonometric interpolating curve to generate curves in the plane. And it is obtained by piecing together segments which are convex combinations of circular arcs or straight line segments [9, 10]. The classical interpolating and smoothing cubic splines requires the recalculation of the entire spline function when a single data point is changed. This can be a severe drawback when implementing real trajectory planning under the presence of unpredictable changes of waypoint, like the appearance of sudden obstacles. Since the trigonometric splines have the property that each of its spline segments depends only on the data in its neighborhood, they can overcome this perceived difficulty. Only four points are used to its computation. If one wants to change a particular interpolation point then at most two segments on the left and two segments on the right have to be computed instead of computing everything from the beginning. Another property of this curve is that it can reduce the computation by selecting the proper time interval $\Delta t$ $(\Delta t \in[0,1])$. Fig. (1) shows the calculation point with various time interval.

The spline segment connecting point $W_{i}($ at $t=0)$ to point $W_{i+1}($ at $t=1)$ is denoted by $S_{i}(t)$ and it is given by following convex combination of two other curves, $L_{i}(t), T_{i}(t)$.

$$
S_{i}(t)=\cos ^{2}(\pi / 2) L_{i}(t)+\sin ^{2}(\pi / 2) T_{i}(t)
$$

Where, $i$ is number of waypoints.

The component $L_{i}(t)$ calculated by using the waypoints $W_{i}, W_{i+1}$ and $W_{i-1}$. If the points $W_{i}$, $W_{i+1}$ and $W_{i-1}$ define a straight line, $\vec{u} \times \vec{v}=0$, then $L_{i}(t)$ is the straight line segment connecting $W_{i}$ (at $t=0$ ) to point $W_{i+1}$ (at $t=1$ ) as shown in Eq. (22). Where, $\vec{u}=W_{i}-W_{i-1}$ and $\vec{v}=W_{i+1}-W_{i}$. 


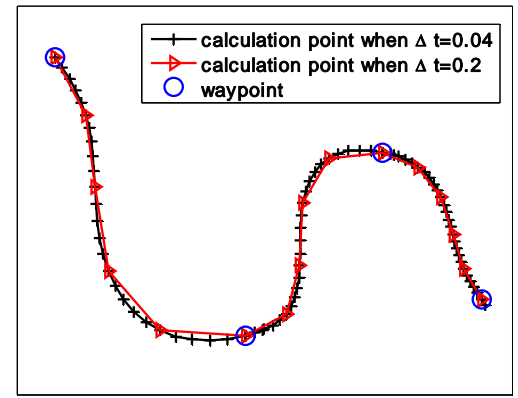

Fig. 1. Calculation point with time interval

Otherwise, consider the circle defined by the three points and let $L_{i}(t)$ be the circular arc, $\vec{u} \times \vec{v} \neq 0$, joining $W_{i}($ at $t=0)$ to point $W_{i+1}$ (at $t=1$ ) that does not contain $W_{i-1}$ and it can be written as Eq. (23).

$$
\begin{gathered}
L_{i}(t)=(1-t) W_{i}+t W_{i+1}, t \in[0,1] \\
L_{i}(t)=m_{L}+\frac{\sin \left(t \theta_{L}\right)}{\sin \left(\theta_{L}\right)} \vec{b}_{L}+\frac{\sin \left((1-t) \theta_{L}\right)}{\sin \left(\theta_{L}\right)} \vec{a}_{L}, t \in[0,1]
\end{gathered}
$$

Where, $m_{L}$ is the center of circle which passes through points $W_{i}, W_{i+1}$ and $W_{i-1}$. The components $\vec{a}_{L}$ and $\vec{b}_{L}$ is defined by $W_{i}-m_{L}, W_{i+1}-m_{L}$, respectively and $\theta_{L}$ is a angle between $\vec{a}_{L}$ and $\vec{b}_{L}$.

The previous algorithm (for $L_{i}(t)$ ), Eq. (22) and Eq. (23), is also implemented to compute $T_{i}(t)$ but it uses the points $W_{i}, W_{i+1}$ and $W_{i+2}$ instead of $W_{i}, W_{i+1}$ and $W_{i-1}$. If the tree point $W_{i}$, $W_{i+1}$ and $W_{i+2}$ are defined straight line, we use Eq. (24). Otherwise, we use Eq. (25).

$$
\begin{gathered}
T_{i}(t)=(1-t) W_{i}+t W_{i+1}, t \in[0,1] \\
T_{i}(t)=m_{T}+\frac{\sin \left(t \theta_{T}\right)}{\sin \left(\theta_{T}\right)} \vec{b}_{T}+\frac{\sin \left((1-t) \theta_{T}\right)}{\sin \left(\theta_{T}\right)} \vec{a}_{T}, t \in[0,1]
\end{gathered}
$$

Where, $m_{T}$ is the center of circle which passes three points. $\vec{a}_{T}=W_{i}-m_{T}, \vec{b}_{L}=W_{i+1}-m_{T}, \theta_{T}$ is a angle between $\vec{a}_{T}$ and $\vec{b}_{T}$.

In this manner, trigonometric interpolating curve is constructed from $n-1$ segments. Each segment is defined as the convex combination of two overlapping circular arcs or line segments using the neighboring points in the data sequence as shown in Fig. (2). For this reason, the construction of the first and last segments requires the extension of the data sequence by the points $W_{0}$ and $W_{n+1}$. In this work we calculate the additional points via the following equation.

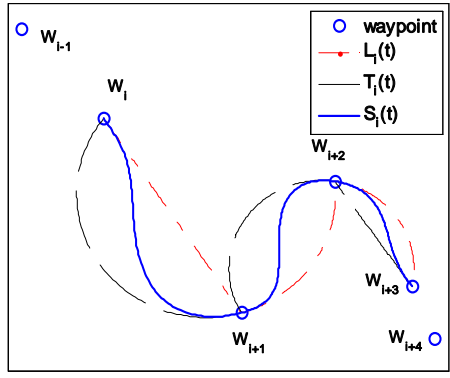

Fig. 2. Trigonometric interpolating curve

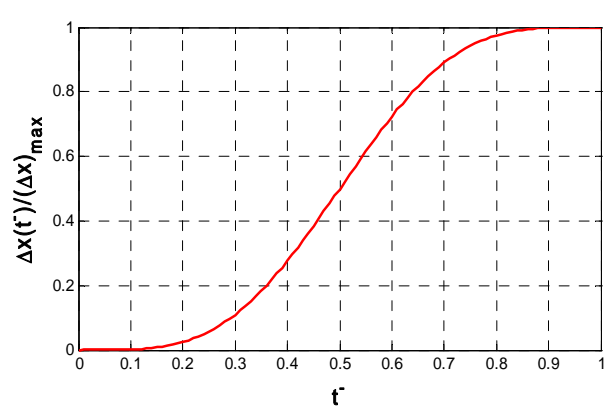

Fig. 3. Description of bob-up/down, acc/de maneuver 


$$
\begin{aligned}
& W_{0}=2 W_{1}-W_{2} \\
& W_{n+1}=2 W_{n}-W_{n-1}
\end{aligned}
$$

In the present study, we define the additional maneuver attributes, bob-up / bob-down maneuver and acceleration / deceleration maneuver, to generate the flight guidance in 3 -dimentsion. The trigonometric functions [2,5] are utilized to define these maneuver trajectory because of its smoothness and simplicity. The height variations of bob-up, bob-down maneuver and forward speed changes of acceleration and deceleration maneuver are described as following formula Eq. (27).

$$
\Delta x(\bar{t})=\frac{(\Delta x)_{\max }}{16.0}[8+\cos (3 \pi \bar{t}-9 \cos (\pi \bar{t})]
$$

Where, $\bar{t}=\left(t-t_{\text {entry }}\right) /\left(t_{\text {finish }}-t_{\text {entry }}\right) \quad 0 \leq \bar{t} \leq 1$

The time, $t_{\text {entry }}$ and $t_{\text {finish }}$, denote the entry and finish time of each maneuver and $(\Delta x)_{\max }$ is the maximum variation in state variables.

\section{Applications}

SDRE technique was applied to the waypoint guidance for the Bo-105 rotorcraft configuration. The quadratic cost function used in this study is defined by penalizing the trajectory deviation from the target trajectory and the additional control effort from the initial trim controls. The quadratic cost function with no terminal cost is implemented and any system constraints are not imposed for the simplicity of analysises.

Where,

$$
\begin{aligned}
f_{C O}\left(\bar{x}_{R}(t), u(t), t\right) & =0.5\left(\bar{x}_{R}-\bar{x}_{t \arg e t}\right)^{T} Q\left(\bar{x}_{R}-\bar{x}_{t \arg e t}\right) \\
& +0.5\left(u-u_{\text {trim }}\right)^{T} R\left(u-u_{\text {trim }}\right)
\end{aligned}
$$

$\bar{x}_{R}$ and $\bar{x}_{\text {target }}$ are reduced rigid body states and target states, respectively.

$$
\begin{aligned}
\bar{x}_{R}(t)= & {\left[u, v, w, p, q, \dot{\psi}, \phi, \theta, \psi, x_{E}, y_{N}, h\right]^{T} } \\
R= & \operatorname{diag}\left(r_{\delta_{0}}, r_{\delta_{1 C}}, r_{\delta_{1 S}}, r_{\delta_{T R}}\right) \\
Q= & \operatorname{diag}\left(q_{u}, q_{v}, q_{w}, q_{p}, q_{q}, q_{r}, q_{\phi},\right. \\
& \left.q_{\theta}, q_{\psi}, q_{x E}, q_{y N}, q_{H}\right)
\end{aligned}
$$

The target states, $\bar{x}_{\text {target }}$, are set to be the trim states except for those which need the description of their time variation for a specific maneuver.

The control weighting matrix, $R$, and the state weighting matrix, $Q$, used in this study consist of the components listed in Table 1, 2, respectively. To verify the SDRE controller through the waypoint guidance simulation, we use the simulation environment which is comprised of three independent PCs.

Table 1. Control weighting matrix

\begin{tabular}{|c|c|c|c|c|}
\hline $\begin{array}{c}\text { Bob-up bob- } \\
\text { down }\end{array}$ & $r_{\delta_{0}}$ & $r_{\delta_{1 C}}$ & $r_{\delta_{1 S}}$ & $r_{\delta_{T R}}$ \\
\hline $\begin{array}{c}\text { Accel. De. } \\
\text { maneuver }\end{array}$ & $1.0 \mathrm{e}+04$ & $3.0 \mathrm{e}+05$ & $5.0 \mathrm{e}+05$ & $2.0 \mathrm{e}+04$ \\
\hline Waypoint tracking & $2.0 \mathrm{e}+04$ & $5.3 \mathrm{e}+05$ & $5.3 \mathrm{e}+05$ & $5.0 \mathrm{e}+04$ \\
\hline
\end{tabular}


Figure 4 shows simulation environment used in this study. The artificial rotorcraft model using the Level 2 rotor model in Ref. [11] is implemented in PC1, which receives control inputs calculated by the SDRE controller in PC2 and sends flight state information to PC2 and PC3. The flight control computer (PC2: FCC) updates the feedback gain on the artificial rotorcraft after solving the SDC factorization and the ARE.

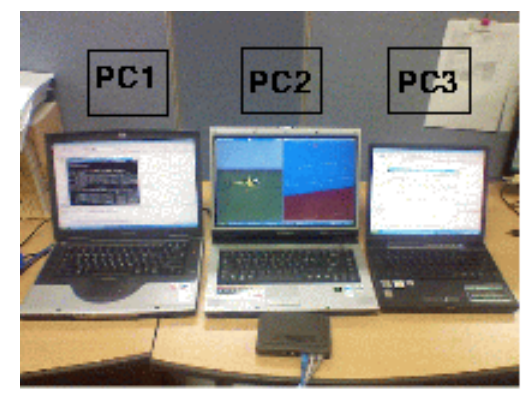

Fig. 4. Simulation environment

Table 2. State weighting matrix

\begin{tabular}{|c|c|c|c|c|c|c|}
\hline & $q_{u}$ & $q_{v}$ & $q_{w}$ & $q_{p}$ & $q_{q}$ & $q_{r}$ \\
\hline bob-up & 100.0 & 50.0 & 5.0 & 100.0 & 30.0 & 100.0 \\
\hline Accel. ' De. maneuver & 80.0 & 40.0 & 10.0 & 60.0 & 30.0 & 30.0 \\
\hline Waypoint tracking & 30.0 & 10.0 & 30.0 & 80.0 & 120.0 & 80.0 \\
\hline & $q_{\phi}$ & $q_{\theta}$ & $q_{\psi}$ & $q_{x E}$ & $q_{y N}$ & $q_{H}$ \\
\hline bob-up & 500.0 & 900.0 & 900.0 & 50.0 & 50.0 & 50.0 \\
\hline Accel. ' De. maneuver & 100.0 & 100.0 & 100.0 & 0.0 & 10.0 & 10.0 \\
\hline Waypoint tracking & 500.0 & 500.0 & 3000.0 & 0.0 & 0.0 & 100.0 \\
\hline
\end{tabular}

Table 3. Waypoints of guidance 1

\begin{tabular}{|c|c|c|}
\hline Waypoints & X position $(\mathrm{m})$ & Y position $(\mathrm{m})$ \\
\hline 1 & 0.0 & 0.0 \\
\hline 2 & 600.0 & 0.0 \\
\hline 3 & 600.0 & 600.0 \\
\hline 4 & 0.0 & 600.0 \\
\hline
\end{tabular}

Table 4. Waypoints of guidance 2

\begin{tabular}{|c|c|c|}
\hline Waypoints & X position $(\mathrm{m})$ & Y position $(\mathrm{m})$ \\
\hline 1 & 0.0 & 0.0 \\
\hline 2 & 700.0 & 0.0 \\
\hline 3 & 360.0 & 940.0 \\
\hline 4 & -410.0 & 300.0 \\
\hline 5 & -580.0 & 770.0 \\
\hline 6 & -1350.0 & 130.0 \\
\hline 7 & -1520.0 & 600.0 \\
\hline 8 & -2250.0 & 0.0 \\
\hline
\end{tabular}


The ground control station (PC3: GCS) identifies the maneuver attributes and generates the switching signal in order for FCC to design the corresponding SDRE controller.

In this paper, the rotorcraft maneuver sequence is like below:

i. Bob-up to $15 \mathrm{~m}$

ii. Acceleration to $10 \mathrm{~m} / \mathrm{s}$

iii. Waypoint guidance

iv. Deceleration to hover

iv. Bob-down at ground

The rotorcraft starts its maneuver from the hovering state at point $(0,0,0)$. First, the height increase to 15 meters during the bob-up maneuver. Next, forward speed isaccelerated up to $10 \mathrm{~m} / \mathrm{s}$ and rotorcraft passes the pre-defined waypoints. And then forward speed is decelerated up to the hover. Finally, the height decrease up to the ground.

To show the effectiveness of the proposed method, we defined two kinds of waypoint guidance. It is generated by series of waypoints listed in Table 3 and Table 4.

Figs. $5 \sim 9$ show the results of waypoint guidance 1. Fig. 5 and Fig. 6 compare the prescribed trajectory generated by using the waypoints in Table 3 with calculated optimal trajectory. The SDRE controller shows some deviation from the target trajectory but the results generally trace the prescribe trajectory well.

Fig. 6 shows the calculated optimal trajectory in 3-dimension. It presents that the rotorcraft go into the waypoint guidance from the bob-up maneuver at point 1 . And after passing through the last point, the simulation finishes with bob-down maneuver.

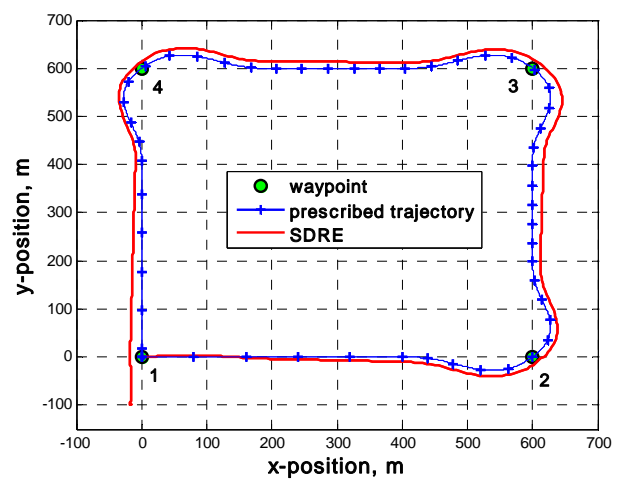

Fig. 5. Maneuver trajectory of guidance 1 in 2-D

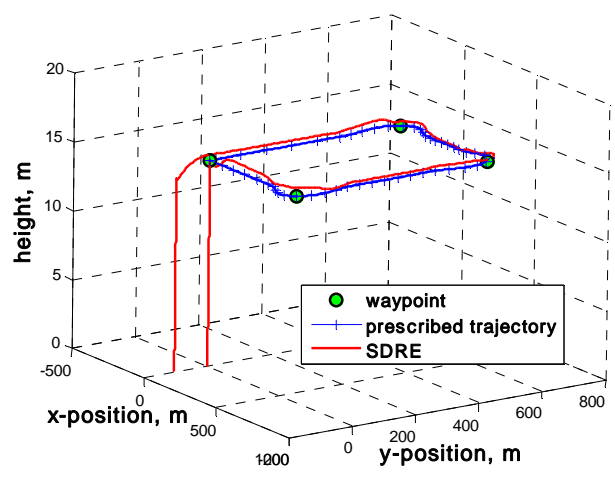

Fig. 6. Maneuver trajectory of guidance 1 in 3-D

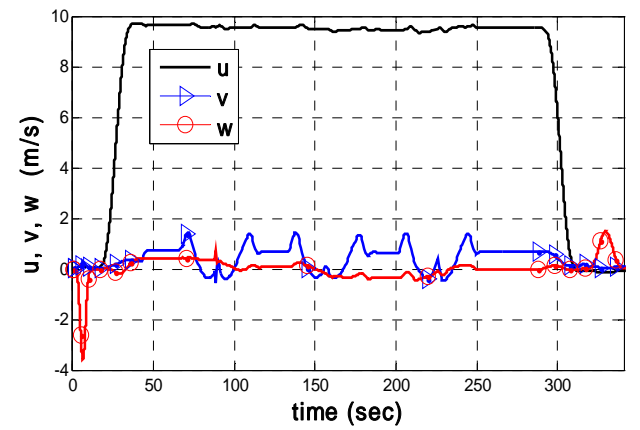

Fig. 7. Velocity components of guidance 1

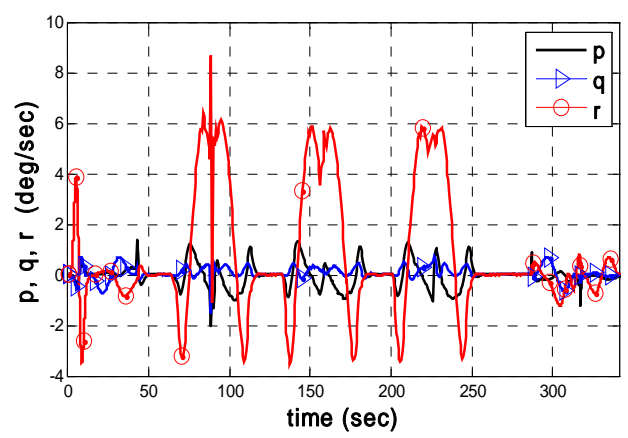

Fig. 8. Roll, pitch and yaw rate of guidance 1 
Figs. 7 9 show the variation of rotorcraft velocity components, angular velocity components and controls. From the Fig. 6, we can fine that the rotorcraft goes into the waypoint guidance through the acceleration of forward speed and then it goes into hover state again after deceleration.

Figs. $10 \sim 14$ show the results of waypoint guidance 2 . The guidance 2 is somehow more aggressive than the guidance 1 but the results generally trace the prescribe trajectory well. Fig. 10 and Fig. 11 compare the prescribed trajectory with calculated optimal trajectory in 2-D and 3-D. And the Figs. 12 14 present also velocity, angular rate and the control variation during the maneuver.

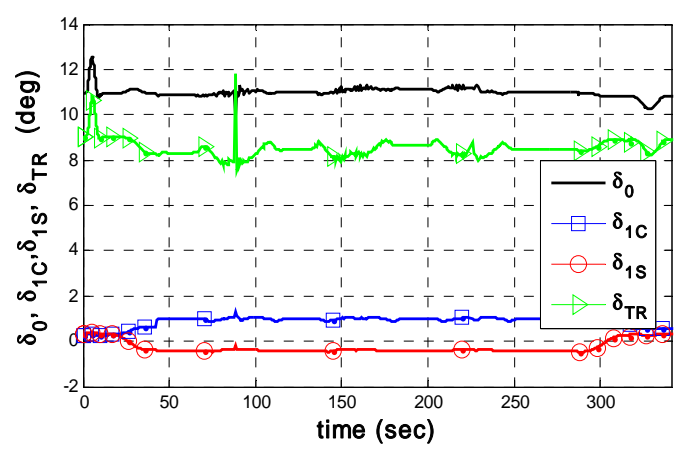

Fig. 9. Control inputs of guidance 1

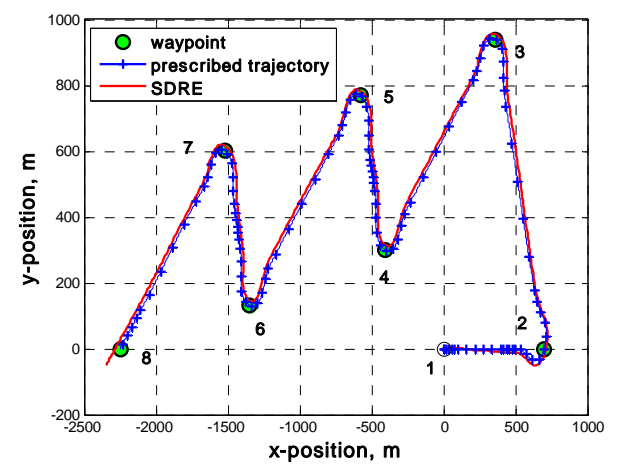

Fig. 10. Maneuver trajectory of guidance 2 in 2-D

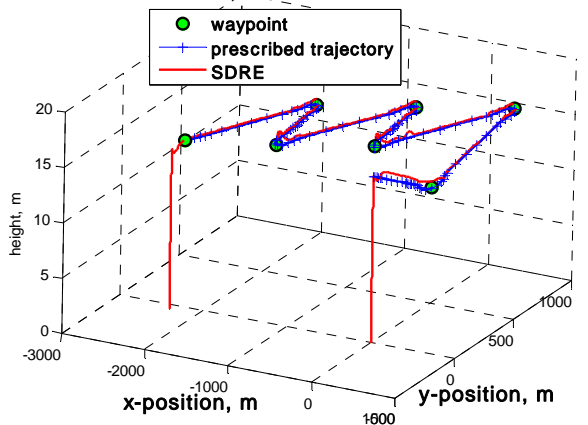

Fig. 11. Maneuver trajectory of guidance 2 in 3-D

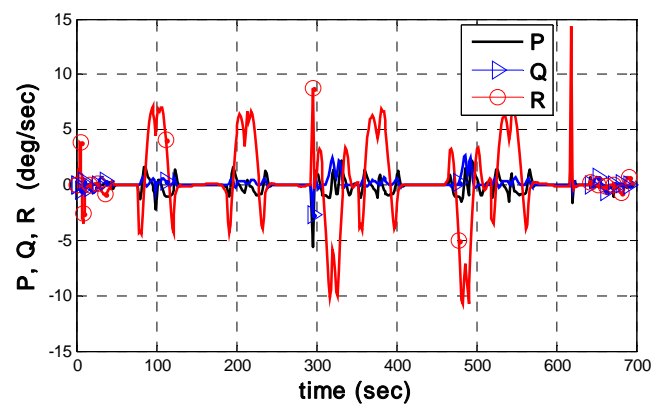

Fig. 13. Roll, pitch and yaw rate of guidance 2

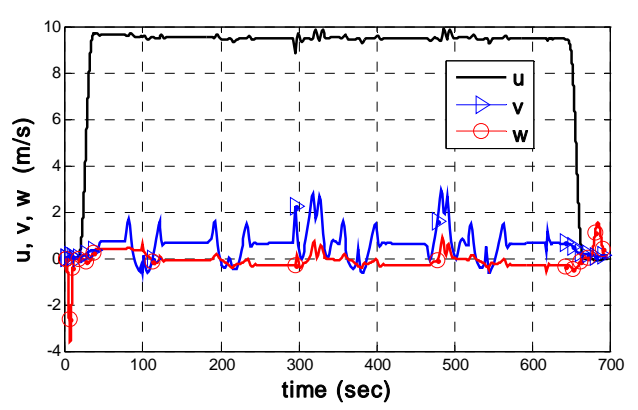

Fig. 12. Velocity components of guidance 2

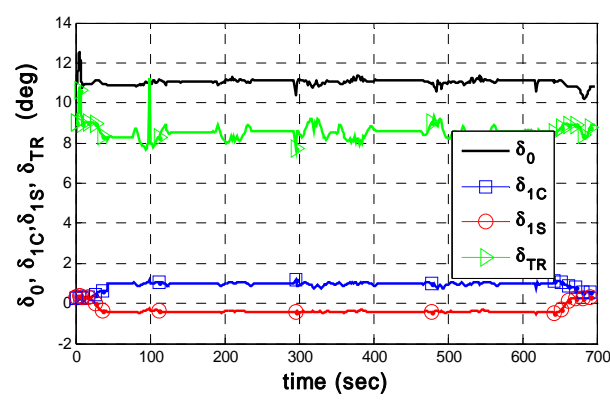

Fig. 14. Control inputs of guidance 2 


\section{Conclusions}

This paper has studied the application method of SDRE technique to the waypoint guidance. The trajectory passing through the series of waypoints has generated by using trigonometric spline. The results show the SDRE controller can be applied to the rotorcraft guidance and control. The controller has been verified using the simulation environment of high fidelity rotorcraft model. After the guidance simulation, the results show the controlled trajectory trace the prescribed it well and indicate the successful implementation of the rotorcraft SDRE controller.

\section{Acknowledgments}

This research was supported by by the Korea Aerospace Research Institute (KARI) under the Korean Helicopter Program (KHP) Dual-Use Core Components Development Program funded by the Ministry of Commerce, Industry, and Energy (MOCIE).

\section{References}

1. James R. Cloutier, 1997, "State-Dependent Riccati Equation Techniques: An Overview", Proceeding of the Anerican Control Conference, New Mexico, pp. 932 936.

2. Chang-Joo Kim, Sang Kyung Sung, Chang Deok Yang, and Yung Hoon Yu, 2008, "Rotorcraft Trajectory Tracking Using the State-Dependent Riccati Equation Controller", Transaction of the Japan Society for Aeronautical and Space Sciences, Vol. 51, No. 173, pp. 184 192.

3. W. Langson, A. G. Alleyne, 1997, "Infinite horizon optimal control for a class of nonlinear systems," Proceedings of the American Control Conference, pp. 3017 3022.

4. P. K. Menon, T. Lam, L. S. Crawford, V. H. L. Cheng, 2002, "Real-Time Computational Method for SDRE Nonlinear Control of Missiles," Proceedings of the American Control Conference, Anchorage, pp. 232 237.

5. Chang-Joo Kim, Sang Kyung Sung, Soo Hyung Park, and Sung Nam Jung, 2008, "Selection of Rotorcraft Models for Application to Optimal Control Problems", Jounal of Guidance, Control, and Dynamics, Vol. 31, No. 5, pp. 1386 1399.

6. Alexander Bogdanov, Eric Wan, Geoff Harvey, 2004, "SDRE Flight Control For XCell and R-Max Autonomous Helicopters", 43rd IEEE Conference on Decision and Control, December 14-17, pp. 1196 1203.

7. Randal W. Beard, Timothy W. McLain, Michael A. Goodrich, 2002, "Coordinated Target Assignment and Intercept for Unmanned Air Vehicles", IEEE Transactions on Robotics and Automation, Vol. 18, No. 6, pp. 911 922.

8. Vedran Bakaric, Zotan Vukic, Radovan Antonic, 2004, "Improved Basic Planar Algorithm of Vehicle Guidance Through Waypoints by the Line of Sight", International Symposium on Control, Communications and Signal Processing, IEEE, pp. 541 544.

9. Nagy M. and T. Vendel, 2000, "Generating curves and swept surfaces by blended circles", Computer Aided Geometric Design, Vol. 17, pp. 197 206.

10. Rodriges, R., F. Leite, and S. Rosa, 2003, "On the generation of a trigonometric interpolating curve in $R^{3}$ ", 11th International Conference on Advanced Robotics, pp. 1629 1634.

11. Chang-Joo Kim, Chul Yong Yun, and Seok Choi, 2005, "Fully Implicit formulation and Its Solution for Rotor Dynamics by Using Differential Algebraic Equation(DAE) Solver and Partial Periodic Trimming Algorithm(PPTA)", 31st European Rotorcraft Forum, Italy.

12. Chang Deok Yang, Min Jae Kim, Jung Hwan Lee, Ji Seung Hong and Chang-Joo Kim, 2008, "Research on the Design of Helicopter Nonlinear Optimal Controller using SDRE Technique", The Korean Society for Aeronautical \& Space Sciences, Vol. 36, No. 12, pp. $1152 \sim 1162$.

13. H. T. Banks and K. Ito, 1985, "A Numerical Algorithm for Optimal Feedback Gains in High Dimensional LQR Problems", NASA CR178207. 